\title{
Nutritional Status of Children with Neurodevelopment Disorders from a Tertiary Medical Center of Bangladesh
}

\author{
Akhter $\mathrm{S}^{\mathrm{a}}$, Shefa $\mathrm{J}^{\mathrm{b}}$, Hasan $\mathrm{MM}^{\mathrm{c}}$
}

\begin{abstract}
Background: Cerebral palsy (CP), CP with epilepsy, epilepsy, speech delay, Down's syndrome, autism, ADHD and vertigo are the most common disabilities among children in Bangladesh. Nutritional status assessment is important as these children are at risk of under nutrition, over nutrition or obesity. The primary objective of this study was to determine the nutritional status of children with neuro-disorder and secondary objective was to find out the factors correlated with poor nutritional status in these children.

Methods: A retrospective study was conducted from a vast number of patients with neurodevelopment disorder attending the out-patient department (OPD) of Institute of Paediatric Neurodisorder and Autism (IPNA), BSMMMU, Dhaka with 149 sample cases from January 2015 to August 2015. Common indicators used to assess the nutrition status of neuro-disorder children were z-scores for weight-for-age, height-for-age, Body Mass Index-for-age percentile.

Results: During the 8 months study period, a total of 149 children were taken aged from 1-180 months where Cerebral palsy (CP), CP with epilepsy, epilepsy, speech delay, Down's syndrome, Autism, ADHD and Vertigo were included and most of the children's nutritional status were $C P$ had moderately impaired nutritional (53\%),CP with epilepsy had mildly impaired nutritional status [(44\%), Epilepsy and Speech delay both disorders had same moderately impaired nutritional status(53\%), Autism had normal nutritional status(40\%), children with Down's syndrome had severely impaired nutritional status(43\%), ADHD children were mostly obese(33\%) \& most of the children with vertigo had moderately impaired nutritional status (67\%).

Conclusions: In a developing country like Bangladesh it could be observed that children with Down's syndrome were at risk of being severely impaired nutritional status, while children with $C P$, epilepsy, speech delay \& vertigo were at risk of being moderately impaired nutritional status and children with ADHD were at risk of being overweight or obese. Children with autism had mostly normal nutritional status, while children of CP with epilepsy were at risk of being mildly impaired nutritional status. Beside low monthly income family with low aged child was somewhere related with nutrition status.
\end{abstract}

Key words: Neurodevelopment Disorders, Cerebral palsy (CP), CP with epilepsy, epilepsy, Speech delay, Down's syndrome, Autism, ADHD, Vertigo, Nutritional Status.

(BIRDEM Med J 2018; 8(2): 118-125)

Author Information

a. Professor Shaheen Akhter, Project Director, Institute of Paediatric Neurodisorder \& Autism (IPNA), Bangabandhu Sheikh Mujib Medical University (BSMMU), Shahabag, Dhaka.

b. Dr. Jannatara Shefa, Research Medical Officer, Institute of Paediatric Neurodisorder \& Autism (IPNA), Bangabandhu Sheikh Mujib Medical University (BSMMU), Shahabag, Dhaka.

c. Md. Mahmudul Hasan, Nutritionist, Institute of Paediatric Neurodisorder \& Autism (IPNA), Bangabandhu Sheikh Mujib Medical University (BSMMU), Shahabag, Dhaka.

Address of correspondence: Dr. Jannatara Shefa, Research Medical Officer, Institute of Paediatric Neurodisorder \& Autism (IPNA), Bangabandhu Sheikh Mujib Medical University (BSMMU), Shahabag, Dhaka. Email: shefaafnan@yahoo.com

Received: January 22, 2018

Accepted: February 28, 2018

\section{Introduction}

Good nutrition is an essential component of good health. Nutritional status has a significant impact on overall health and quality of life in children with neurodevelopment disabilities; both under and over nutrition generally lead to increased health care use and reduced participation in educational and social activities. Malnutrition is frequently associated with impairment of linear growth, reduced peripheral circulation and wound healing, increased spasticity and irritability. The overall prevalence of malnutrition in neurological impairment children is difficult to estimate, due to the heterogeneity of neurological disorders. 
Neurological disorder is a complex condition because it varies according to age, gender, socioeconomic status, cultural background, geographical area and health condition $^{1}$. The degree of disability for any individual with disability also depends upon the relationship between various environmental factors. ${ }^{2}$

Chronic diseases have a significant impact on nutritional status. In neurologically impaired (NI) patients, malnutrition negatively affects quality of life and is associated with increased health care use and impaired participation in various activities. ${ }^{3}$ Nutritional rehabilitation has been associated with improved overall health, improved peripheral circulation, healing of decubitus ulcers, decreased spasticity, decreased irritability and improved gastroesophageal reflux in patients with neuro-developmental disabilities. ${ }^{4-7} \mathrm{~A}$ significant proportion of children with neurodevelopmental disabilities are undernourished. ${ }^{8-11}$ This state of malnutrition may have been once considered to be part of the disease, but there is now a better recognition of the importance of nutrition in this population. Nutritional care of children with neurodevelopmental disabilities has improved with the advent of less invasive enteral access methods and better tolerated enteral formulas. According to the United Nations Children's Fund (UNICEF), it was estimated that the number of children with disabilities under 18 years of age was approximately 150 million. ${ }^{12}$ This included children with autism spectrum disorders (ASD), cerebral palsy (CP), Down syndrome (DS), Epilepsy, Speech Delay (SD) learning disabilities and other types of disabilities. We need to provide these often complicated patients with appropriate nutritional support by involving a multidisciplinary team of physicians, nurses, dietitians, speech and occupational therapists, and psychologists working to improve the child's and the family's quality of life.

There are no studies on nutritional status of children with neuro-disorder in our set up, the aim of this study was to evaluate the nutritional status and factors correlated with malnutrition amongst neuro-disorder children in out-patient department of IPNA, BSMMU between the ages of 1 month to 15 years. It was hoped that the study will promote interest in the nutritional status of these unfortunate.

\section{Methods}

A retrospective study was conducted from a vast number of patients with neurodevelopment disorder attending the out-patient department (OPD) of Paediatric Neurodisorder and Autism (IPNA), BSMMU, Dhaka with 149 sample cases from January 2015 to August 2015. The consecutive sampling method was utilized. All children with neurodevelopment disorder who met the inclusion criteria and whose parents/guardians gave consent during the study period were recruited. Clearance was obtained from the Hospital Ethical Committee of BSMMU and informed written consent was obtained from each parent or guardian at the time of interview.

Children with a diagnosis of neurodevelopment disorder made by medical doctor confirmed by patient records attending this center, aged between 1 month and 15 years, were consecutively recruited for the study. Patients with any other known chronic ailments that would independently affect nutritional status were excluded from the study.

Data collection took place in a side nutritional counseling room within the ward. A structured, pretested questionnaire was administered to the accompanying parent or caregiver to collect the bio-demographic data of patients.

Each child's weight was measured to use room scale. Children who could not stand were weighed in the arms of the mother. The difference between the combined weight of mother and child and that of mother alone was recorded as the child's weight. Height was measured using a Stadiometer for patients aged more than 2 years who were able to stand flat-footed and straight. The recumbent length of participants less than 2 years of age and those who though 2 years or more were unable to stand erect was measured using a Stadiometer with a fixed head rest and a movable foot board.

All measurements were performed by the first author using the same instrument throughout the study. The anthropometric data were analyzed using the current WHO Growth chart (2006). Height and weight were used to classify the children into different types of malnutrition. Malnutrition was categorized using the WHO classification (2006).

The data was subjected to statistical analysis using the SPSS IBM 20 software package. Chi square test was 
used to determine associations and $\mathrm{P}<0.05$ were taken as significant.

\section{Ethical consideration}

The study was conducted after getting approval from the ethical committee of the BSMMU Hospital. All children were recruited to the study only after explaining to the parents/guardians about the study and after the parents/guardians signed the consent form. All information obtained from the study was kept confidential.

\section{Results}

In total, 149 children with neuro-disorders were recruited into the study. The results in table showed that the majority (56\%) of the Children belong to were from the age group of 0-36 months. A large percentage of the parents belong to were from the age group and occupation of 31-40 years (51\%), Service holder (Father) and 21-30 years (50\%), House wife (mother) respectively (Table-I).

Table I. Socio-demographic status of respondent $(\mathrm{N}=149)$

\begin{tabular}{llcc}
\hline Variables & Age group & Frequency & Percent \\
\hline Children's age & $0-36$ & 84 & 56 \\
& $37-48$ & 8 & 5 \\
& $49-60$ & 17 & 11 \\
Father's age & $61 \&$ above & 40 & 27 \\
& $21-30$ & 36 & 24 \\
& $31-40$ & 76 & 51 \\
Mother's age & $40 \&$ above & 37 & 25 \\
& $<20$ \& 20 & 20 & 13 \\
& $21-30$ & 74 & 50 \\
Occupation category & $31-40$ & 49 & 33 \\
Father's & Day labour & 13 & 9 \\
occupation & Service holder & 71 & 48 \\
& Business & 51 & 34 \\
Mother's & Home abroad & 14 & 9 \\
occupation & House wife & 136 & 91 \\
\hline
\end{tabular}

The education level of the parents shows that $30 \%$ of father had completed their graduation and above, 37\% of mothers were below Secondary School Certificate (SSC) level (Table-II)

Table II. Level of education

Education Father's education Mother's education level Frequency Percentage Frequency Percentage

\begin{tabular}{lllll}
\hline Primary & 32 & 21 & 22 & 15 \\
Below SSC & 35 & 23 & 55 & 37 \\
SSC-HSC & 38 & 25 & 40 & 27 \\
Graduation & 44 & 30 & 32 & 21 \\
\& above & & & & \\
\hline
\end{tabular}

Among the total respondents, Majority $(85 \%)$ of the respondent were no allergic reaction to any food beside most of the respondent (70\%) were not avoided any food during at first $0-4$ months pregnancy period. During full period of pregnant time, A large percentage (88\%) of the respondents were not suffered any diseases. Approximately $44 \%$ of the participants were completed Exclusive Breast Feeding (EBF) (0-6 months) rest 56\% were not completed (Table-III).

Table III. Pregnancy history and breast feeding of the respondent

During pregnancy Present/ Frequency Percentages history Absent

At first 0-4 months of pregnant period

Allergic reaction to $\quad$ Yes $23 \quad 15$

$\begin{array}{lll}\text { any food } & \text { No } & 126\end{array}$

$\begin{array}{llll}\text { Avoid any food } & \text { Yes } & 45 & 30\end{array}$

No $\quad 104 \quad 70$

Full period of pregnancy

$\begin{array}{llll}\text { Suffered any diseases } & \text { Yes } & 18 & 12\end{array}$

No $\quad 131 \quad 88$

After delivery

Child completed EBF Yes $\quad 65 \quad 44$

No $\quad 84 \quad 56$

The figure is clearly evident that $53 \%$ of the patients with neuro-disorder were male and $47 \%$ were female (Figure 1). 

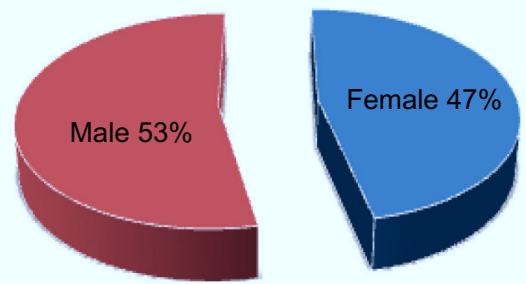

Figure 1. Male-female distribution of the children

The study shows that Majority (94\%) of the patients was Muslim rest of others were from the Hindu and Buddha (Figure-2).

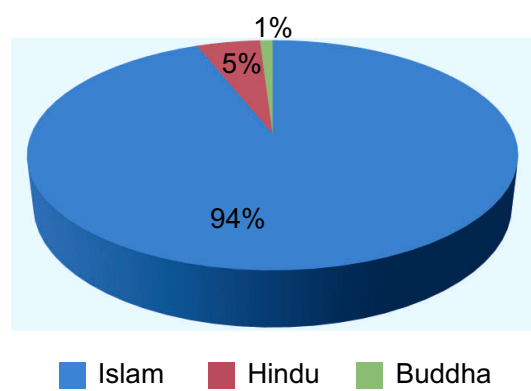

Figure 2. Distribution of the respondents by Religion

Among the total participant, a majority $(70 \%)$ of the patients were birth by caesarian delivery beside minority $(30 \%)$ were home delivery (Figure 3 ).

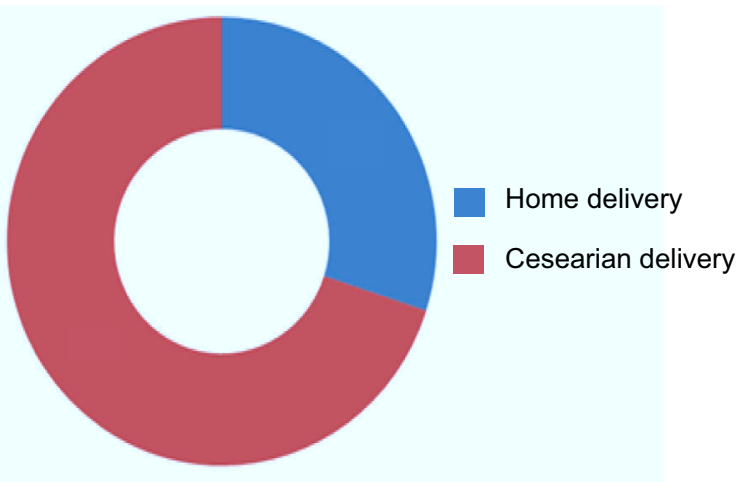

Figure 3. Distribution according to mood of delivery

The study revealed that most of the respondents lived in urban $(56 \%)$, whereas others lived in semi urban $(33 \%)$ and rural (11\%) (Figure 4).

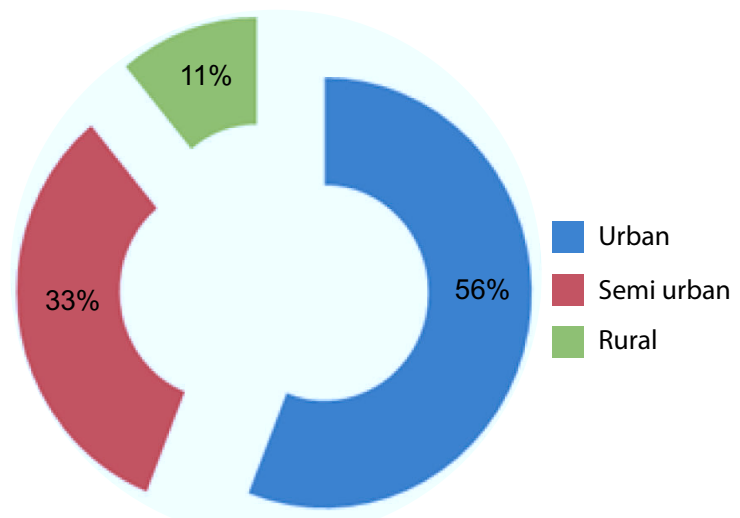

Figure 4. Distribution according to residence

Concerning about correlation between nutritional status and independent variable

The results in Figure 5-IXdemonstrate the correlation with nutrition status were not found in allergy, food avoidance in pregnancy and mother educational background in somewhere it was mildly correlated with monthly family income and low child age.

Correlation with monthly income and nutritional status In case of below 10,000Tk. Income more children were moderately and severely impaired nutrition status where as 11,000-25,000 income range highest number of children were moderately and severely impaired nutrition status (Figure-5).

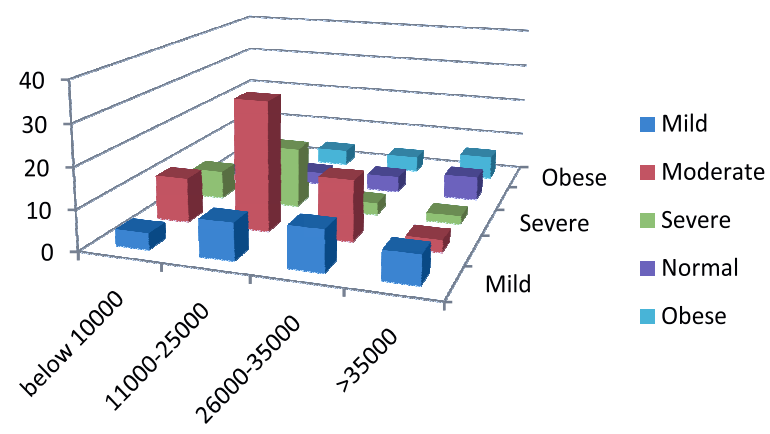

Figure 5. Correlation with monthly income and nutritional status

\section{Correlation with NDD child's ages and nutritional status}

It could be observed that in $0-36$ month's age range moderately deprived nutrition status children were more. In 36-48 months age range obese were more where as 
in 48-60 months age range moderately and severely deprived nutrition status common. But in 60 and above months age range moderately deprived nutrition status more (Figure 6).

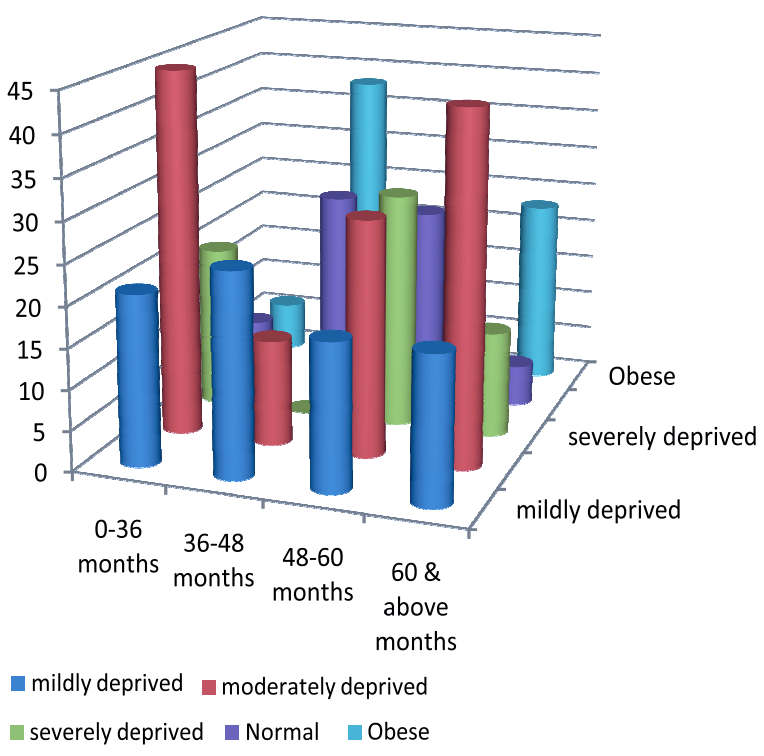

Figure 6. Correlation with NDD child's ages and nutritional status

Correlation with food avoidance and nutritional status in pregnancy

There were almost no correlation with nutrition status and avoidance of food in pregnancy (Figure 7).

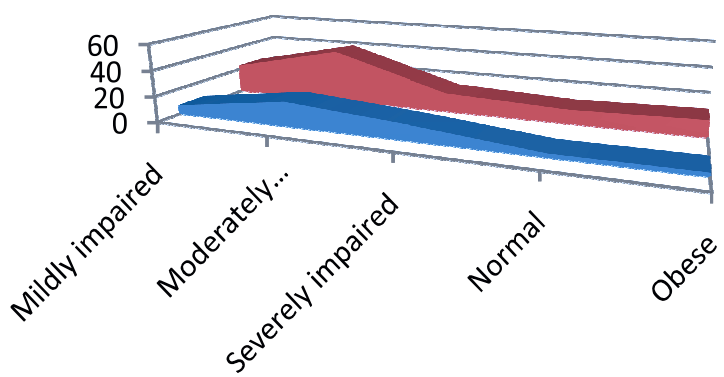

Avoid food during pregnancy Don't avoid

Figure 7. Correlation with food avoidance and nutritional status
Correlation with having or not food allergy of mother during pregnancy and nutritional status.

Nutrition status and food allergy during pregnancy were not associated with each other in this study (Figure 8).

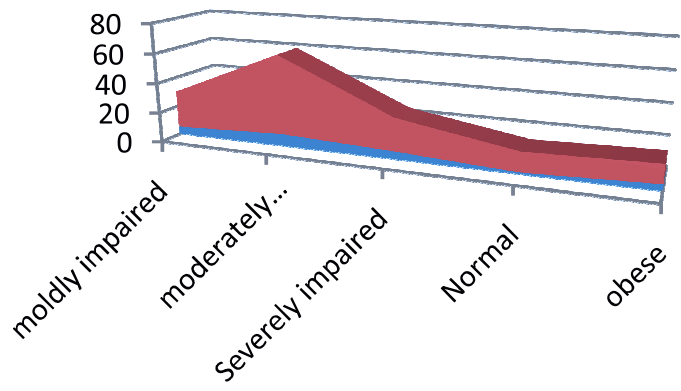

Don't have allergy
Food allergy during pregnancy

Figure 8. Correlation with having or not food allergy of mother during pregnancy and nutritional status

Correlation with mother's education and nutritional status

Mother educational background could not effect the nutrition status of the children in this study (Figure-9).

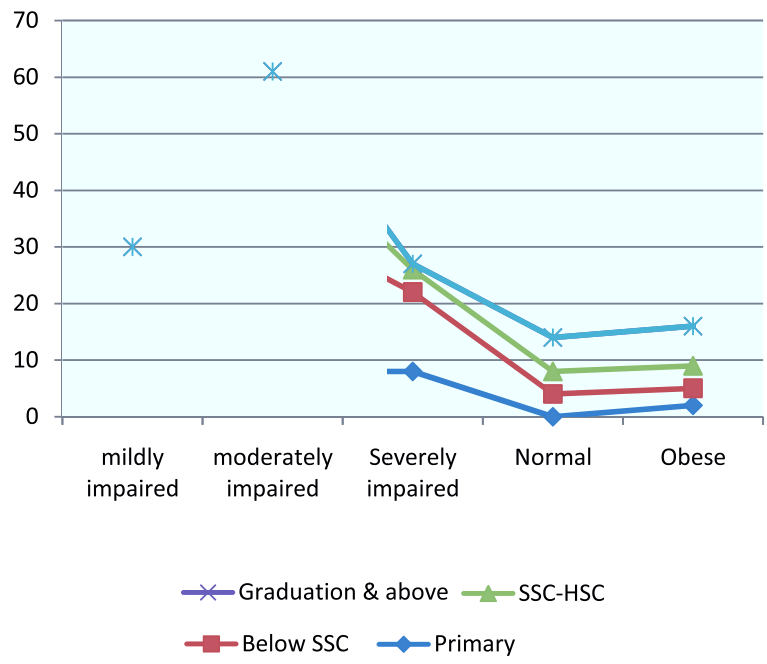

Figure 9. Correlation with mother's education and nutritional status

Nutritional status of different types of NDD children

Overall,the study found that children with Down's syndrome were severely impaired nutritional 
status(43\%) and at risk of being underweight, while children with ASD were normal nutritional status(40\%) and children with $\mathrm{CP}$ tend to be at risk of being moderately impaired. Different types of neurodevelopment disorder children had different types of nutritional status impairment (Figure-10).

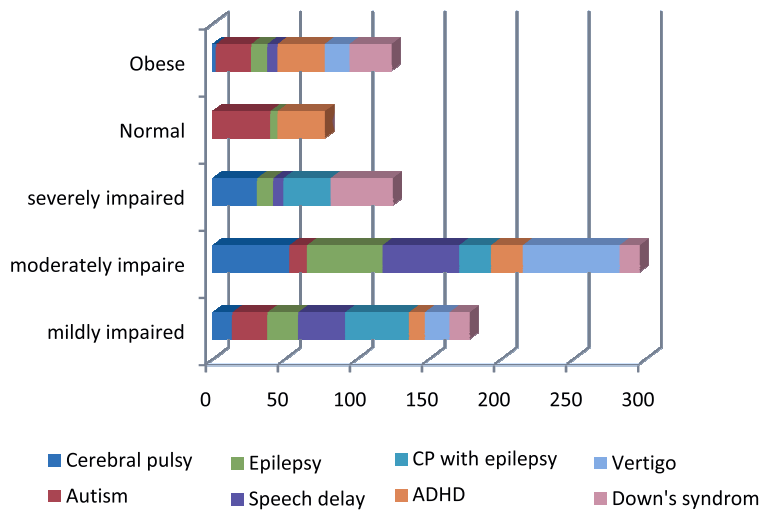

Figure 10. Nutritional status of different types of NDD children

\section{Down syndrome}

The study has shown that children with DS were shorter in height than the standard references of typically developing children, so they had severely impaired nutritional status( $43 \%)$.

\section{Cerebral palsy and $\mathrm{CP}$ with Epilepsy}

This study reported that a large proportion of children with $\mathrm{CP}$ had moderately impaired nutritional status $(53 \%)$ on the other handCP with epilepsy were mildly impaired nutritional status(44\%).

\section{Epilepsy}

Thestudyinvestigatedthenutritional statusof children CP with epilepsy were mildly impaired nutritional status(44\%) while Epilepsy were moderately impaired nutritional status $(53 \%)$

\section{Autism spectrum disorders}

According to the study investigated the nutritional status of children with ASD children were normal nutritional status $(40 \%)$ and their tend to be overweight or obese as compared to the general population.

\section{ADHD}

The nutritional status of children with ADHD were at risk of being overweight or obese whereas nutrition status of child with ASD was normal nutritional status $(40 \%)$

\section{Speech Delay}

Although Speech delay is the common symptom of ASD children, in this study nutritional status of children with ASD children were normal but only children with speech delay were at risk of being moderately impaired nutritional status.

\section{Discussion}

Nutritional status is an important indicator of the overall health status and wellbeing of children with neurodisorder. It portrays the physical and mental growth of children and whether they are at risk of being underweight, overweight or obese. This study sought to assess the nutritional status and factors associated with malnutrition among children with neurodisorder who were attended to at BSMMU hospital outpatient child neurology center using anthropometries measurements.

The impact of poor nutritional status and poor growth among children with disability may eventually lead to poor motor function, bone health, social participation and healthcare utilisation ${ }^{13}$.For example, the consequences of overweight and obesity problems in children with DS are the increased risk of developing adult obesity and non-communicable diseases such as diabetes and hypertension ${ }^{14}$.Malnutrition among children with neurodevelopment disorder can lead to poor growth and nutrient deficiencies ${ }^{15}$. However, evidence on the nutritional status of children with DS, CP, and ASD, ADHD, Epilepsy, Speech Delay is still limited and little is known on the overall prevalence of the nutritional status of these children, including the methods, tools and indicators to assess this.Various tools may be required to assess the nutritional status of children with disability due to the disparities in their health conditions, growth and development stage.Good knowledge and understanding are required among health care professionals in order to monitor the health status of these children, so that further treatment or intervention could be planned for this group.

In total, 149 children with Neuro-disorder were recruited into the study. The above table show that Majority $(56 \%)$ of the Children belong to were from the age group of 0 36 months.Majority of the parents belong to were from the age group and occupation of 31 to 40 years 
(51\%), Service holder (Father) and 21 to 30 years $(50 \%)$,House wife (Mother) respectively. Among the parents, $30 \%$ of father had completed their Graduation and above, $37 \%$ of mothers were below SSC level. Among the total respondents,Majority ( $85 \%)$ of the respondent were no allergic reaction to any food beside most of the respondent $(70 \%)$ were not avoided any food during at first 0-4 months pregnant time. During full period of pregnant time, Majority of the respondents $(88 \%)$ were not suffered any diseases. Approximately $44 \%$ of the participants were completed Exclusive Breast Feeding (0-6 months) beside 56\% were not completed.

In case of below10,000Tk.income more children were moderately and severely impaired nutrition status where as 11,000-25,000 income range highest number of children were moderately and severely impaired nutrition status. It could be observed that in0-36 months age range moderately deprived nutrition status children were more. In 36-48 months age range obese were more where as in 48-60 months age range moderately and severely deprived nutrition status common But in 60 and above months age range moderately deprived nutrition status more. Overall, the study found that children with Down's syndrome were severely impaired nutritional status( $43 \%)$ and at risk of being underweight, while children with ASD were normal nutritional status (40\%) and children with $\mathrm{CP}$ tend to be at risk of being moderately impaired. The study has shown that children with DS were shorter than the standard references of typically developing children, so they had severely impaired nutritional status (43\%). This study reported that a large proportion of children with $\mathrm{CP}$ had moderately impaired nutritional status $(53 \%)$ beside $\mathrm{CP}$ with epilepsy were mildly impaired nutritional status (44\%). The study investigating the nutritional status of children CP with epilepsy were mildly impaired nutritional status(44\%) while Epilepsy were moderately impaired nutritional status (53\%). According to the study investigating the nutritional status of children with ASD children were normal nutritional status $(40 \%)$ and their tend to be overweight or obese as compared to the general population. The nutritional status of children with ADHD were at risk of being overweight or obese while nutrition status of children with ASD were normal nutritional status(40\%).Although Speech delay is the common symptom of ASD children, to this study informed us nutritional status of children with ASD children were normal but only children with speech delay were at risk of being moderately impaired nutritional status.

Therefore, the present review will explore evidence on the nutritional status among children with neurodisability, particularly among children with DS, CP, Epilepsy and ASD. It is hoped that these findings will support future research on the nutritional status for children with disability and the future planning of health interventions for this population.

\section{Conclusion}

In conclusion, there is emerging evidence on the nutritional status of children with ASD, DS, CP, CP with Epilepsy, Speech Delay, Vertigo and ADHD, although this is still very limited in developing countries including Bangladesh. Findings of this scoping review demonstrated that children with Down syndrome were at risk of severely impaired nutritional status, while children with CP, Epilepsy, Speech delay and vertigo were at risk of being moderately impaired nutritional status and children with ADHD were at risk of being overweight or obese.Children with Autism had mostly normal nutritional status, while children of $\mathrm{CP}$ with Epilepsy were at risk of being mildly impaired nutritional status beside low monthly income with low aged child was somewhere related with nutrition status.

\section{Recommendations}

- Nutritional support should be an integral part in the management of neuro-development disorder children.

- Nutritional problems of children with neurodevelopmental disorder should be identified early and screened regularly with a multidisciplinary team for malnutrition.

- Excessive intake should be avoided to become obese.

- Parents should be educated on nutrition for the betterment of the child's health.

- Government should take proper steps for improving nutrition of neuro-development disorder children.

- Large scale studies should be needed to improve the overall situation.

Conflict of interest: Nothing to declare. 


\section{References}

1. Tan K, Yadav H. Assessing the development of children with disability in Malaysia, Medical Journal of Malaysia 2008; 63: 199-202. PubMed Abstract

2. Colver A, A shared framework and language for childhood disability, Developmental Medicine and Child Neurology, 2005; 780-784. PubMed Abstract | Publisher Full Text | Google Scholar

3. Samson-Fang L, Fung E, Stallings VA, et al. Relationship of nutritional status to health and societal participation in children with cerebral palsy. J Pediatr 2002;141:637-43. [PubMed]

4. Patrick J, Boland M, Stoski D, Murray GE. Rapid correction of wasting in children with cerebral palsy. Dev Med Child Neurol 1986;28:734-39. [PubMed]

5. Shapiro BK, Green P, Krick J, Allen D, Capute AJ. Growth of severely impaired children:Neurological versus nutritional factors. Dev Child Neurol 1986;28:729-33. [PubMed]

6. Sanders KD, Cox K, Cannon R, et al. Growth response to enteral feeding by children with cerebral palsy. J Parenter Enteral Nutr 1990;14:23-26. [PubMed]

7. Lewis D, Khoshoo V, Pencharz PB, Golladay ES. Impact of nutritional rehabilitation on gastroesophageal reflux in neurologically impaired children. J Pediatr Surg. 1994;29:167-79. [PubMed]

8. Krick J, Murphy-Miller P, Zeger S, Wright E. Pattern of growth in children with cerebral palsy. J Am Diet Assoc 1996;96:680 85. [PubMed]

9. Stallings VA, Charney EB, Davies JC, Cronk CE. Nutritional status and growth of children with diplegic or hemiplegic cerebral palsy. Dev Med Child Neurol 1993;35:997-1006. [PubMed]

10. Stallings VA, Cronk CE, Zemel BS, Charney EB. Body composition in children with spastic quadriplegic cerebral palsy. J Pediatr 1995;126:833-39. [PubMed]

11. Stallings VA, Charney EB, Davies JC, Cronk CE. Nutritionrelated growth failure of children with quadriplegic cerebral palsy. Dev Med Child Neurol 1993;35:126-38. [PubMed]

12. United Nations Children's Fund (UNICEF), The state of the world's children: Children with disabilities (2013), UNICEF. New York.

13. Caglia $\mathrm{P}$, Luca $\mathrm{S}$ et al. Enteral nutrition in patients with chronic neurological diseases. Minerva Gastroenterology Dietol 2000; 46:199-20.

14. Chandra RK, Kumari S. Nutrition and immunity. J Nutr. 1994; 124:1433S-1435S.

15. Engsner G, Habre D, Sjogren I, Vahlquist B: Brain growth in children with kwashiorkor. ActaPaediatr Scand 1974; 63: 687-94. 